\title{
THE MAXIMUM MODULUS PRINCIPLE FOR CR FUNCTIONS
}

\author{
ANDREI IORDAN
}

\begin{abstract}
Let $M$ be a CR submanifold of $\mathbf{C}^{\prime \prime}$ without extreme points. Then, the modulus of any $\mathrm{CR}$ function on $M$ cannot have a strong local maximum at any point of $M$.
\end{abstract}

Preliminaries. Let $M$ be a smooth manifold embedded as a locally closed real submanifold of $\mathbf{C}^{n}$. We denote by $\bar{\partial}_{M}$ the tangential Cauchy-Riemann operator on $M$ induced by the Cauchy-Riemann operator $\bar{\partial}$ on $\mathbf{C}^{n}$ and with $\operatorname{HT}_{p}(M)$ the holomorphic tangent space to $M$ at a point $p \in M$.

Let us recall some of the definitions and results of [4].

DEFINITION 1. $\bar{\partial}_{M}$ obeys the local maximum modulus principle on $M$ if given any open connected set $U$ in $M$ and any $u$ differentiable in $U$ such that $\bar{\partial}_{M} u=0$ on $U$, then $u$ cannot have a (weak) local maximum at any point of $U$ unless $u$ is constant on $U$.

Definition 2. We call a point $p \in M$ an extreme point of $M$ if there exists a local holomorphic coordinate system $z=\left(z_{1}, \ldots, z_{n}\right)$ in a neighborhood $U$ of $p$ such that $z(p)=0$ and $M \cap U \subset\left\{z \mid y_{1} \geqslant 0\right\}$. Here we assume that locally near $p, M$ is not contained in any $\mathbf{C}^{k}$ for $k<n$.

Definition 3. (i) For any $p \in M$ and $X \in \mathrm{HT}_{p}(M)$ set $Z=X-i Y$, where $Y=J X \in \mathrm{HT}_{p}(M)$ and $J$ is the multiplication with $(-1)^{1 / 2}$ which defines the complex structure on $\mathbf{R}^{2 n}$.

The Levi form at $p$ assigns to $Z$ the normal vector $L_{p}(Z)$ defined by $L_{p}(Z)=$ $B_{p}(X, X)+B_{p}(Y, Y)$, where $B_{p}$ is the second fundamental form of $M$ at $p$.

(ii) We denote $N_{p}(M)$ as the normal space of $M$ at $p$.

For any $\xi \in N_{p}(M)$ the map $L_{p}^{\xi}$ defined by $L_{p}^{\xi}(Z)=\left\langle L_{p}(Z), \xi\right\rangle$ is called the Levi form of $M$ at $p$ in the $\xi$ direction. Here $\langle$,$\rangle represents the real inner product in$ $\mathbf{R}^{2 n}$.

We assume that $p=0$ and $\operatorname{codim}_{\mathbf{R}} M=q$. Then in a neighborhood $U$ of the origin there are smooth real functions $\rho_{1}, \ldots, \rho_{q}$ such that $d \rho_{1} \wedge \cdots \wedge d \rho_{q \mid 0} \neq 0$ and

$$
M \cap U=\left\{z \in U \mid \rho_{1}(z)=\cdots=\rho_{q}(z)=0\right\} .
$$

We may assume that $d \rho_{1}(0), \ldots, d \rho_{q}(0)$ are orthonormal.

Received by the editors September 27, 1984 and, in revised form, March 13, 1985.

1980 Mathematics Subject Classification. Primary 32F25. 
If $\xi \in N_{0}(M)$, then $\xi=\sum_{i=1}^{q} \xi_{i} d \xi_{i}(0)$ and if $Z=\sum_{j=1}^{n} w_{j}\left(\partial / \partial z_{j}\right) \in \mathrm{HT}_{0}(M)$, then $\sum_{j=1}^{n}\left(\partial \rho_{i} / \partial z_{j}\right)(0) w_{j}=0$ for $1 \leqslant i \leqslant q$.

In these conditions

$$
L_{0}^{\xi}(Z)=-4 \sum_{i, j, k} \xi_{i} \frac{\partial^{2} \rho_{i}}{\partial z_{j} \partial \bar{z}_{k}}(0) w_{j} \bar{w}_{k}
$$

In [4] the following results are proved.

Proposition 1. If $p$ is an extreme point of $M$, then there exists a normal direction $\xi \in N_{p}(M)$ such that $L_{p}^{\xi}$ is positive definite:

Proposition 2. If for a point $p \in M$ there exists $\xi \in N_{p}(M)$ such that $L_{p}^{\xi}$ is strictly positive definite, then $p$ is an extreme point of $M$.

THEOREM 1. If $\bar{\partial}_{M}$ obeys the local maximum modulus principle on $M$, then $M$ can contain no extreme point.

In [4], it is conjectured that the converse of Theorem 1 is also true.

Statement of results. A submanifold $M$ of $\mathbf{C}^{n}$ is called a CR manifold if $\operatorname{dim}_{C} \mathrm{HT}_{p}(M)$ is constant on $M$.

We say that $M$ has CR dimension $m$ if $\operatorname{dim}_{\mathbf{C}} \mathrm{HT}_{p}(M)=m$, and we denote $\mathrm{CR} \operatorname{dim}(M)=m$.

A totally real submanifold of $\mathbf{C}^{n}$ is a $\mathrm{CR}$ submanifold of $\mathbf{C}^{n}$ with $\mathrm{CR} \operatorname{dim}(M)=0$.

A complex valued smooth function $f$ on $M$ for which $\bar{\partial}_{M} f=0$ on $M$ is called a CR function on $M$.

THEOREM 2. Each point of a totally real submanifold $M \subset \mathbf{C}^{n}$ is an extreme point of $M$.

THEOREM 3. If $M$ is a $C R$ submanifold of $\mathbf{C}^{n}$ without extreme points, then for any $C R$ function $f$ on $M,|f|$ cannot have a strong local maximum at any point of $M$.

Proof of Theorem 2. We know from [5] that there exists a nonnegative function $\varphi \in C^{2}\left(\mathbf{C}^{n}\right)$ strictly plurisubharmonic in a neighborhood $D$ of $M$ such that

$$
M=\{z \in D \mid \varphi(z)=0\}=\{z \in D \mid \operatorname{grad} \varphi=0\} .
$$

Let $p \in M$. We assume that $p=0$ and in a neighborhood $V$ of $p$ we have $V \cap M=\left\{z \in V \mid \rho_{1}(z)=\cdots=\rho_{q}(z)=0\right\}$ with $d \rho_{1} \wedge \cdots \wedge d \rho_{q \mid 0} \neq 0$.

Let $\rho=\varphi+\varepsilon \rho_{1} \in C^{2}(V)$, where $\varepsilon>0$ is chosen small enough such that the complex Hessian of $\rho$ is strictly positive definite at the origin.

We have also $d \rho(0)=\varepsilon d \rho_{1}(0) \neq 0$ and we may assume that $\left(\partial \rho_{1} / \partial z_{1}\right)(0) \neq 0$, so $\left(\partial \rho / \partial z_{1}\right)(0) \neq 0$.

Because $\rho(0)=0$ in a neighborhood of the origin we have:

$$
\begin{aligned}
\rho(z)= & 2 \operatorname{Re} \sum_{i=1}^{n} \frac{\partial \rho}{\partial z_{i}}(0) z_{i}+\operatorname{Re}\left(\sum_{i, j=1}^{n} \frac{\partial^{2} \rho}{\partial z_{i} \partial z_{j}}(0) z_{i} z_{j}\right) \\
& +\sum_{i, j=1}^{n} \frac{\partial^{2} \rho}{\partial z_{i} \partial \bar{z}_{j}}(0) z_{i} \bar{z}_{j}+O\left(|z|^{3}\right) .
\end{aligned}
$$


We make the holomorphic change of coordinates in $\mathbf{C}^{n}$ :

$$
z_{1}^{\prime}=2 i \sum_{i=1}^{n} \frac{\partial \rho}{\partial z_{i}}(0) z_{i}+i \sum_{i, j=1}^{n} \frac{\partial^{2} \rho}{\partial z_{i} \partial z_{j}}(0) z_{i} z_{j}, \quad z_{i}^{\prime}=z_{i} \text { for } 2 \leqslant i \leqslant n .
$$

In the new coordinates we have

$$
\rho\left(z^{\prime}\right)=-y_{1}^{\prime}+\sum_{i, j=1}^{n} a_{i j} z_{i}^{\prime} \bar{z}_{j}^{\prime}+O\left(\left|z^{\prime}\right|^{3}\right)
$$

with $\sum_{i, j=1}^{n} a_{i j} z^{\prime} z_{j}^{\prime}$ strictly positive definite.

Define $S=\{z \in V \mid \rho(z)=0\}$.

It follows that $S \subset\left\{z^{\prime} \mid y_{1}^{\prime} \geqslant 0\right\}$ in the neighborhood of the origin and because $M \cap V \subset S$ the theorem is proved.

Proof of Theorem 3. We shall use the following lemma [2]:

LemMA 1. Let $\Omega$ be an open subset of $R^{N}$ with coordinates $x_{1}, \ldots, x_{N}$. Let $F \in C^{\infty}(\Omega)$ and $L$ be a compact subset of $\Omega$. Suppose that $F(x)<\max _{L} F$ for each $x \in \Omega-L$. Then for any open set $\Omega_{1}$ with $L \subset \Omega_{1} \subset \Omega$, there exists a point $y \in \Omega_{1}$ such that the Hessian of $F$ at $y$ is strictly negative definite.

We denote $d=\operatorname{dim}_{R} M, q=2 n-d$ and $m=\mathrm{CR} \operatorname{dim} M$.

Let us suppose that there exists a CR function $f$ on $M$ such that $|f|$ has a point of strong local maximum. Then there exists a compact set $K \subset M$ such that $\max _{K}|f|$ $>\max _{\partial K}|f|$. We may assume that $K$ is contained in an open set in $R^{d}$ which is part of the atlas that defines $M$. We may assume also that $\max _{K} \operatorname{Re} f>\max _{\partial K} \operatorname{Re} f$. Let us denote $\operatorname{Re} f=\varphi$.

By Lemma 1 there is $p \in K$ such that $\left(\left(\partial^{2} \varphi / \partial t_{i} \partial t_{j}\right)(p)\right)_{1 \leqslant i \leqslant d .1 \leqslant j \leqslant d}$ is strictly negative definite for any real coordinates $\left(t_{1}, \ldots, t_{d}\right)$ in a neighborhood of $p$.

From Theorem 2, we obtain that $m \geqslant 1$. We denote $s=d-2 m$ and $r=m-(d$ $-n)$.

After a complex linear change of coordinates in $\mathbf{C}^{n}, M$ may be represented in the neighborhood of the point $p$ by the equations

$$
\begin{array}{ll}
z_{j}=x_{j}+i g^{j}(x, w), & j=1, \ldots, s, \\
z_{j+s}=h^{j}(x, w), & j=1, \ldots, r, \\
z_{j+s+r}=w_{j}, & j=1, \ldots, m,
\end{array}
$$

where $p$ corresponds to the origin and $\left\{g^{j}\right\}_{j=1, \ldots, s},\left\{h^{j}\right\}_{j=1, \ldots, r}$ are real and complex valued functions respectively vanishing to second order at the origin [7].

Therefore, if

$$
\begin{gathered}
\rho_{1}=y_{1}-g^{1}(x, z), \ldots, \rho_{s}=y_{s}-g^{s}(x, z), \\
\rho_{s+1}=x_{s+1}-\operatorname{Re} h^{1}(x, z), \\
\rho_{s+2}=y_{s+1}-\operatorname{Im} h^{1}(x, z), \ldots, \rho_{q}=y_{s+r}-\operatorname{Im} h^{r}(x, z),
\end{gathered}
$$


where $x=\left(x_{1}, \ldots, x_{s}\right)$ and $z=\left(z_{s+r+1}, \ldots, z_{n}\right)$, then in a neighborhood of the origin we have

$$
\begin{gathered}
M=\left\{z \mid \rho_{1}(z)=\cdots=\rho_{q}(z)=0\right\}, \\
d \rho_{1} \wedge \cdots \wedge d \rho_{q \mid 0} \neq 0 \text { and } d \rho_{1}(0), \ldots, d \rho_{q}(0) \text { are orthonormal. }
\end{gathered}
$$

$\varphi$ is the real part of a CR function so $(\partial \bar{\partial})_{b} \varphi=0[3]$.

If $\tilde{\varphi}$ is a real extension of $\varphi$, then in a neighborhood $U$ of $p$ in $\mathbf{C}^{n}$ we obtain

$$
\partial \bar{\partial} \tilde{\varphi}=\sum_{k=1}^{q} a_{k} \rho_{k}+\sum_{k=1}^{q} b_{k} \partial \rho_{k}+\sum_{k=1}^{q} c_{k} \bar{\partial} \rho_{k}+\sum_{k=1}^{q} d_{k} \partial \bar{\partial} \rho_{k}
$$

with $a_{k} \in C_{(1,1)}^{\infty}(U), b_{k} \in C_{(0,1)}^{\infty}(U), c_{k} \in C_{(1,0)}^{\infty}(U)$ and $d_{k} \in C^{\infty}(U)$ or,

$$
\frac{\partial^{2} \tilde{\varphi}}{\partial z_{i} \partial \bar{z}_{j}}=\sum_{k=1}^{q} a_{i j k} \rho_{k}+\sum_{k=1}^{q} b_{k j} \frac{\partial \rho_{k}}{\partial z_{i}}+\sum_{k=1}^{q} c_{k i} \frac{\partial \rho_{k}}{\partial \bar{z}_{j}}+\sum_{k=1}^{q} d_{k} \frac{\partial^{2} \rho_{k}}{\partial z_{i} \partial \bar{z}_{j}} .
$$

We choose an extension $\tilde{\varphi}$ of $\varphi$ which is independent of the variables $y_{1}, \ldots, y_{s}$, $x_{s+1}, y_{s+1}, \ldots, x_{s+r}, y_{s+r}$ which are normal to $M$ over the origin.

Let $Z \in \mathrm{HT}_{0}(M)$, i.e.

$$
Z=\sum_{i=1}^{n} z_{i}\left(\frac{\partial}{\partial z_{i}}\right)_{0} \text { and } \sum_{i=1}^{n} z_{i} \frac{\partial \rho_{k}}{\partial z_{i}}(0)=0
$$

$k=1, \ldots, q$.

From (2) we obtain

$$
\sum_{i, j=1}^{n} \frac{\partial^{2} \tilde{\varphi}}{\partial z_{i} \partial \bar{z}_{j}}(0) z_{i} \bar{z}_{j}=\sum_{i, j=1}^{n} \sum_{k=1}^{q} d_{k}(0) \frac{\partial^{2} \rho_{k}}{\partial z_{i} \partial \bar{z}_{j}}(0) z_{i} \bar{z}_{j} .
$$

But, with the functions $\rho_{k}$ given by (1), $Z \in \mathrm{HT}_{0}(M)$ if $z_{1}=\cdots=z_{s+r}=0$.

It follows that

$$
\sum_{i, j=1}^{n} \frac{\partial^{2} \tilde{\varphi}}{\partial z_{i} \partial \bar{z}_{j}}(0) z_{i} \bar{z}_{j}=\sum_{i, j=1}^{m} \frac{\partial^{2} \varphi}{\partial w_{i} \partial \bar{w}_{j}}(0) w_{i} \bar{w}_{j}
$$

which is strictly negative definite.

Indeed supposing that

$$
\sum_{i, j=1}^{m} \frac{\partial^{2} \varphi}{\partial w_{i} \partial \bar{w}_{j}}(0) w_{i} \bar{w}_{j}
$$

is not strictly negative definite, by making a complex linear change of coordinates $w_{1}, \ldots, w_{m}$, in the new coordinates $w_{1}^{\prime}, \ldots, w_{m}^{\prime}$ we may assume

$$
\frac{\partial^{2} \varphi}{\partial w_{1}^{\prime} \partial \bar{w}_{1}^{\prime}}(0) \geqslant 0 \text {. }
$$

But

$$
\frac{\partial^{2} \varphi}{\partial w_{1}^{\prime} \partial \bar{w}_{1}^{\prime}}(0)=\frac{1}{4}\left(\frac{\partial^{2} \varphi}{\partial u_{1}^{\prime 2}}(0)+\frac{\partial^{2} \varphi}{\partial v_{1}^{\prime 2}}(0)\right)
$$


where $w_{1}^{\prime}=u_{1}^{\prime}+i v_{1}^{\prime}$ and this contradicts the fact that the real Hessian of $\varphi$ at 0 is strictly negative definite.

Taking the real parts in (3) we obtain

$$
\sum_{i, j=1}^{n} \frac{\partial^{2} \tilde{\varphi}}{\partial z_{i} \partial \bar{z}_{j}}(0) z_{i} \bar{z}_{j}=\sum_{k=1}^{q} \operatorname{Re} d_{k}(0)\left(\sum_{i, j=1}^{n} \frac{\partial^{2} \rho_{k}}{\partial z_{i} \partial \bar{z}_{j}}(0) z_{i} \bar{z}_{j}\right) \text {. }
$$

Hence if $\xi=\sum_{k=1}^{q}\left(-\operatorname{Re} d_{k}(0)\right) d \rho_{k}(0) \in N_{0}(M)$ it follows that $L_{p}^{\xi}$ is strictly positive definite and by Proposition $2, p$ is an extreme point of $M$, which contradicts the fact that $M$ has no extreme points.

Remark. Professor Hugo Rossi pointed to me that Theorem 3 can be proved by using the approximation theorem of Baouendi and Trèves [1] and the techniques from his paper [6].

ACKNOWLEDGEMENT. I wish to thank the referee for his suggestions which simplified the proof of Theorem 3 .

\section{REFERENCES}

1. M. S. Baouendi and F. Trèves, A property of the functions and distributions annihilated by a locally integrable system of complex vector fields, Ann. of Math. (2) 113 (1981), 387-421.

2. R. Basener, Nonlinear Cauchy-Riemann equations and q-pseudoconvexity, Duke Math. J. 43 (1976), 203-215.

3. E. Bedford, $(\partial \bar{\partial})_{h}$ and the real parts of $C R$ functions, Indiana Univ. Math. J. 29 (1980), 333-340

4. D. Ellis, C. D. Hill and C. Seabury, The maximum modulus principle I. Necessary conditions, Indiana Univ. Math. J. 25 (1976), 709-715.

5. F. R. Harvey and R. O. Wells, Jr., Holomorphic approximation and hyperfunction theory on a $C^{1}$ totally real submanifold of a complex manifold, Math. Ann. 197 (1972), 287-318.

6. H. Rossi, Holomorphically convex sets in several complex variables, Ann. of Math. (2) 74 (1961), $470-493$.

7. R. O. Wells, Jr., Holomorphic hulls and holomorphic convexity of differentiable submanifolds, Trans. Amer. Math. Soc. 132 (1968), 245-262.

Institute of Mathematics, 14 ACADemiei Street, 70109 Bucharest, România 